\title{
SOME PROPERTIES OF (AMPLY) G-RADICAL SUPPLEMENTED MODULES
}

\author{
BERNA KOŞAR AND AYTEN PEKIN
}

Received 23 June, 2020

\begin{abstract}
In this work, some new properties of (amply) g-radical supplemented modules are investigated. It is proved that every factor module and every homomorphic image of an amply g-radical supplemented module are amply g-radical supplemented. Let $M$ be a $\pi$-projective and g-radical supplemented module. Then $M$ is amply g-radical supplemented. Let $M$ be a projective and g-radical supplemented module. Then every finitely $M$-generated module is amply g-radical supplemented. Let $R$ be any ring. Then ${ }_{R} R$ is g-radical supplemented if and only if every finitely generated $R$-module is amply g-radical supplemented.
\end{abstract}

2010 Mathematics Subject Classification: 16D10; 16D70

Keywords: g-small submodules, radical, g-supplemented modules, g-radical supplemented modules

\section{INTRODUCTION}

Throughout this paper all rings will be associative with identity and all modules will be unital left modules.

Let $R$ be a ring and $M$ be an $R$-module. We will denote a submodule $N$ of $M$ by $N \leq M$. Let $M$ be an $R$-module and $N \leq M$. If $L=M$ for every submodule $L$ of $M$ such that $M=N+L$, then $N$ is called a small submodule of $M$ and denoted by $N \ll M$. Let $M$ be an $R$-module and $N \leq M$. If there exists a submodule $K$ of $M$ such that $M=N+K$ and $N \cap K=0$, then $N$ is called a direct summand of $M$ and it is denoted by $M=N \oplus K$. A submodule $N$ of an $R$-module $M$ is called an essential submodule of $M$, denoted by $N \unlhd M$, if $K=0$ for every $K \leq M$ with $K \cap N=0$. Let $M$ be an $R$-module and $K$ be a submodule of $M . K$ is called a generalized small (briefly, $g$-small) submodule of $M$ if for every $T \unlhd M$ with $M=K+T$ implies that $T=M$, this is written by $K \ll_{g} M$ (in [14], it is called an $e$-small submodule of $M$ and denoted by $K \ll_{e} M$ ). Let $M$ be an $R$-module. $M$ is called a hollow module if every proper submodule of $M$ is small in $M . M$ is called a local module if $M$ has the largest submodule, i. e. a proper submodule which contains all other proper submodules. Let $U$ and $V$ be submodules of $M$. If $M=U+V$ and $V$ is minimal with respect to this property, or equivalently, $M=U+V$ and $U \cap V \ll V$, then $V$ is called a supplement 
of $U$ in $M . M$ is called a supplemented module if every submodule of $M$ has a supplement in $M$. Let $M$ be an $R$-module and $U, V \leq M$. If $M=U+V$ and $M=U+T$ with $T \unlhd V$ implies that $T=V$, or equivalently, $M=U+V$ and $U \cap V \ll g V$, then $V$ is called a $g$-supplement of $U$ in $M . M$ is said to be $g$-supplemented if every submodule of $M$ has a g-supplement in $M$. Let $M$ be an $R$-module and $U \leq M$. If for every $V \leq M$ such that $M=U+V, U$ has a supplement (g-supplement) $V^{\prime}$ in $M$ with $V^{\prime} \leq V$, then we say $U$ has ample supplements ( $g$-supplements) in $M$. If every submodule of $M$ has ample supplements (g-supplements) in $M$, then $M$ is called an amply supplemented ( $g$-supplemented) module. The intersection of all maximal submodules of an $R$-module $M$ is called the radical of $M$ and denoted by Rad $M$. If $M$ have no maximal submodules, then we denote $\operatorname{Rad} M=M . M$ is said to be semilocal if $M / \operatorname{Rad} M$ is semisimple, i. e. every submodule of $M / \operatorname{Rad} M$ is a direct summand of $M / \operatorname{Rad} M$. Let $M$ be an $R$-module and $U, V \leq M$. If $M=U+V$ and $U \cap V \leq R a d V$, then $V$ is called a generalized (radical) supplement (briefly, Rad-supplement) of $U$ in $M . M$ is said to be generalized (radical) supplemented (briefly, Rad-supplemented) if every submodule of $M$ has a Rad-supplement in $M$. Let $M$ be an $R$-module and $U \leq M$. If for every $V \leq M$ such that $M=U+V, U$ has a Rad-supplement $V^{\prime}$ in $M$ with $V^{\prime} \leq V$, then we say $U$ has ample generalized (radical) supplements (briefly, ample Rad-supplements) in $M$. If every submodule of $M$ has ample Rad-supplements in $M$, then $M$ is called an amply generalized (radical) supplemented (briefly, amply Rad-supplemented) module. The intersection of all essential maximal submodules of an $R$-module $M$ is called the generalized radical (briefly, $g$-radical) of $M$ and denoted by $\operatorname{Rad}_{g} M$ (in [14], it is denoted by $\operatorname{Rad}_{e} M$ ). If $M$ have no essential maximal submodules, then we denote $\operatorname{Rad}_{g} M=M$. $M$ is said to be $g$-semilocal if $M / \operatorname{Rad}_{g} M$ is semisimple, i. e. every submodule of $M / \operatorname{Rad}_{g} M$ is a direct summand of $M / \operatorname{Rad}_{g} M$. Let $M$ be an $R$-module. We say submodules $X$ and $Y$ of $M$ are $\beta^{*}$ equivalent, $X \beta^{*} Y$, if and only if $Y+K=M$ for every $K \leq M$ such that $X+K=M$ and $X+T=M$ for every $T \leq M$ such that $Y+T=M$. We say submodules $X$ and $Y$ of $M$ are $\beta_{g}^{*}$ equivalent, $X \beta_{g}^{*} Y$, if and only if $Y+K=M$ for every $K \unlhd M$ such that $X+K=M$ and $X+T=M$ for every $T \unlhd M$ such that $Y+T=M$. Let $M$ be $R$-module and $X \leq Y \leq M$. If $Y / X \ll M / X$, then we say $Y$ lies above $X$ in $M$.

More information about (amply) supplemented modules are in [2, 6, 13]. More informations about $\mathrm{g}$-small submodules and (amply) g-supplemented modules are in $[4,8,11]$. The definition of (amply) generalized supplemented modules and some properties of them are in [12]. The definition of g-semilocal modules and some properties of them are in [5]. The definition of $\beta^{*}$ relation and some results of this relation are in [1]. The definition of $\beta_{g}^{*}$ relation and some results of this relation are in [10].

Lemma 1. Let $M$ be an R-module. The following assertions hold.

(1) For every $m \in \operatorname{Rad}_{g} M, R m \ll_{g} M$.

(2) If $N \leq M$, then $\operatorname{Rad}_{g} N \leq \operatorname{Rad}_{g} M$.

(3) $\operatorname{Rad}_{g} M=\sum_{L \ll_{g} M} L$. 
Proof. See [3, Lemma 2 and Lemma 3].

\section{G-RADICAL SUPPLEMENTED MODULE}

Definition 1. Let $M$ be an $R$-module and $U, V \leq M$. If $M=U+V$ and $U \cap V \leq$ $\operatorname{Rad}_{g} V$, then $V$ is called a g-radical supplement of $U$ in $M$. If every submodule of $M$ has a g-radical supplement in $M$, then $M$ is called a g-radical supplemented module. $($ See $[3,7]$.)

Clearly we can see that every g-supplemented module is g-radical supplemented. But the converse is not true in general. Every Rad-supplemented module is g-radical supplemented.

Proposition 1. Let $M$ be an $R$-module and $\operatorname{Rad}_{g} V=V \cap \operatorname{Rad}_{g} M$ for every $V \leq M$. Then $M$ is $g$-radical supplemented if and only if $M$ is $g$-semilocal.

Proof. $(\Longrightarrow)$ Clear from [3, Theorem 1].

$(\Longleftarrow)$ Let $U \leq M$. Since $M$ is $\mathrm{g}$-semilocal, $\left(U+\operatorname{Rad}_{g} M\right) / \operatorname{Rad}_{g} M$ is a direct summand of $M / \operatorname{Rad}_{g} M$. By this, there exists $V / \operatorname{Rad}_{g} M \leq M / \operatorname{Rad}_{g} M$ such that $\frac{U+\operatorname{Rad}_{g} M}{\operatorname{Rad}_{g} M} \oplus \frac{V}{\operatorname{Rad}_{g} M}=\frac{M}{\operatorname{Rad}_{g} M}$. Then $\frac{U+\operatorname{Rad}_{g} M}{\operatorname{Rad}_{g} M}+\frac{V}{\operatorname{Rad}_{g} M}=\frac{M}{\operatorname{Rad}_{g} M}$ and $\frac{U+\operatorname{Rad}_{g} M}{\operatorname{Rad}_{g} M} \cap \frac{V}{\operatorname{Rad}_{g} M}=0$. Here $\frac{M}{\operatorname{Rad}_{g} M}=\frac{U+\operatorname{Rad}_{g} M}{\operatorname{Rad}_{g} M}+\frac{V}{\operatorname{Rad}_{g} M}=\frac{U+\operatorname{Rad}_{g} M+V}{\operatorname{Rad}_{g} M}=\frac{U+V}{\operatorname{Rad}_{g} M}$ and $0=\frac{U+\operatorname{Rad}_{g} M}{\operatorname{Rad}_{g} M} \cap \frac{V}{\operatorname{Rad}_{g} M}=$ $\frac{\left(U+\operatorname{Rad}_{g} M\right) \cap V}{\operatorname{Rad}_{g} M}=\frac{U \cap V+\operatorname{Rad}_{g} M}{\operatorname{Rad}_{g} M}$. Since $\frac{U+V}{\operatorname{Rad}_{g} M}=\frac{M}{\operatorname{Rad}_{g} M}, U+V=M$. Since $\frac{U \cap V+\operatorname{Rad}_{g} M}{\operatorname{Rad}_{g} M}=0$, $U \cap V+\operatorname{Rad}_{g} M=\operatorname{Rad}_{g} M$ and $U \cap V \leq \operatorname{Rad}_{g} M$. By $U \cap V \leq V$ and $\operatorname{Rad}_{g} V=$ $=V \cap \operatorname{Rad}_{g} M, U \cap V \leq V \cap \operatorname{Rad}_{g} M=\operatorname{Rad}_{g} V$. Hence $V$ is a g-radical supplement of $U$ in $M$ and $M$ is g-radical supplemented.

Proposition 2. Let $M$ be an $R$-module and $R a d V=V \cap R a d M$ for every $V \leq M$. Then $M$ is Rad-supplemented if and only if $M$ is semilocal.

Proof. ( $\Longrightarrow)$ Clear from [12, Proposition 2.6 (2)].

$(\Longleftarrow)$ Let $U \leq M$. Since $M$ is semilocal, $(U+\operatorname{Rad} M) / \operatorname{Rad} M$ is a direct summand of $M / \operatorname{Rad} M$. By this, there exists $V / \operatorname{Rad} M \leq M / \operatorname{Rad} M$ such that $\frac{U+\operatorname{Rad} M}{\operatorname{Rad} M} \oplus \frac{V}{\operatorname{Rad} M}=$ $\frac{M}{\operatorname{Rad} M}$. Then $\frac{U+\operatorname{Rad} M}{\operatorname{Rad} M}+\frac{V}{\operatorname{Rad} M}=\frac{M}{\operatorname{Rad} M}$ and $\frac{U+\operatorname{Rad} M}{\operatorname{Rad} M} \cap \frac{V}{\operatorname{Rad} M}=0$. Here $\frac{M}{\operatorname{Rad} M}=\frac{U+\operatorname{Rad} M}{\operatorname{Rad} M}+$ $\frac{V}{\operatorname{Rad} M}=\frac{U+\operatorname{Rad} M+V}{\operatorname{Rad} M}=\frac{U+V}{\operatorname{Rad} M}$ and $0=\frac{U+\operatorname{Rad} M}{\operatorname{Rad} M} \cap \frac{V}{\operatorname{Rad} M}=\frac{(U+\operatorname{Rad} M) \cap V}{\operatorname{Rad} M}=\frac{U \cap V+\operatorname{Rad} M}{\operatorname{Rad} M}$. Since $\frac{U+V}{\operatorname{Rad} M}=\frac{M}{\operatorname{Rad} M}, U+V=M$. Since $\frac{U \cap V+\operatorname{Rad} M}{\operatorname{Rad} M}=0, U \cap V+\operatorname{Rad} M=\operatorname{Rad} M$ and $U \cap V \leq \operatorname{Rad} M$. By $U \cap V \leq V$ and $\operatorname{Rad} V=V \cap \operatorname{Rad} M, U \cap V \leq V \cap \operatorname{Rad} M=\operatorname{Rad} V$. Hence $V$ is a Rad-supplement of $U$ in $M$ and $M$ is Rad-supplemented.

Lemma 2. Let $X \beta_{g}^{*} Y$ in $M, Y$ be a g-radical supplement of $U$ in $M$ and $U \unlhd M$. Then $U \cap X \leq \operatorname{Rad}_{g} M$.

Proof. Since $Y$ is a g-radical supplement of $U$ in $M, M=U+Y$ and $U \cap Y \leq$ $\operatorname{Rad}_{g} Y \leq \operatorname{Rad}_{g} M$. Since $M=U+Y$ and $U \unlhd M$ and $X \beta_{g}^{*} Y, M=U+X$. Let $T$ be any essential maximal submodule of $M$. Here $U \cap Y \leq \operatorname{Rad}_{g} M \leq T$. Assume 
that $U \cap X \not \leq T$. Then $U \cap X+T=M$ and since $M=U+X$, by [2, Lemma 1.24], $X+U \cap T=M$. Since $U \unlhd M$ and $T \unlhd M, U \cap T \unlhd M$. Since $X \beta_{g}^{*} Y, Y+U \cap T=M$ and since $U+T=M$, by [2, Lemma 1.24] again, $U \cap Y+T=M$. Then by $U \cap Y \leq T$, $M=U \cap Y+T=T$. This is a contradiction. Hence $U \cap X \leq T$ for every essential maximal submodule $T$ of $M$ and $U \cap X \leq \operatorname{Rad}_{g} M$.

Corollary 1. Let $X \beta^{*} Y$ in $M, Y$ be a g-radical supplement of $U$ in $M$ and $U \unlhd M$. Then $U \cap X \leq \operatorname{Rad}_{g} M$.

Proof. Clear from Lemma 2.

Corollary 2. Let $X$ lies above $Y, Y$ be a g-radical supplement of $U$ in $M$ and $U \unlhd M$. Then $U \cap X \leq \operatorname{Rad}_{g} M$.

Proof. Clear from Lemma 2.

Definition 2. Let $M$ be an $R$-module and $V \leq M$. If $V$ is a g-radical supplement of an essential submodule in $M$, then $V$ is called an eg-radical supplement submodule (briefly, eg-radical supplement) in $M$.

Lemma 3. Let $M$ be an $R$-module. If every submodule of $M$ is $\beta_{g}^{*}$ equivalent to an eg-radical supplement in $M$, then $M$ is g-semilocal.

Proof. Let $X / \operatorname{Rad}_{g} M \leq M / \operatorname{Rad}_{g} M$. Since $X \leq M$, by hypothesis, there exists an eg-radical supplement $Y$ in $M$ such that $X \beta^{*} Y$. Since $Y$ is an eg-radical supplement in $M$, there exists $U \unlhd M$ such that $Y$ is a g-radical supplement of $U$ in $M$. By Lemma 2, $U \cap X \leq \operatorname{Rad}_{g} M$. Since $X \beta_{g}^{*} Y$ and $U \unlhd M$ and $Y+U=M, X+U=$ $M$. Then $\frac{M}{\operatorname{Rad}_{g} M}=\frac{X+U}{\operatorname{Rad}_{g} M}=\frac{X}{\operatorname{Rad}_{g} M}+\frac{U+\operatorname{Rad}_{g} M}{\operatorname{Rad}_{g} M}$ and $\frac{X}{\operatorname{Rad}_{g} M} \cap \frac{U+\operatorname{Rad}_{g} M}{\operatorname{Rad}_{g} M}=\frac{X \cap\left(U+\operatorname{Rad}_{g} M\right)}{\operatorname{Rad}_{g} M}=$ $\frac{U \cap X+\operatorname{Rad}_{g} M}{\operatorname{Rad}_{g} M}=\frac{\operatorname{Rad}_{g} M}{\operatorname{Rad}_{g} M}=0$. Hence $\frac{M}{\operatorname{Rad}_{g} M}=\frac{X}{\operatorname{Rad}_{g} M} \oplus \frac{U+\operatorname{Rad}_{g} M}{\operatorname{Rad}_{g} M}$ and $M / \operatorname{Rad}_{g} M$ is semisimple. Thus $M$ is $\mathrm{g}$-semilocal.

Corollary 3. Let $M$ be an R-module. If every submodule of $M$ lies above an eg-radical supplement in $M$, then $M$ is g-semilocal.

Proof. Clear from Lemma 3.

\section{AMPLY G-RADICAL SUPPLEMENTED MODULES}

Definition 3. Let $M$ be an $R$-module and $U \leq M$. If for every $V \leq M$ such that $M=U+V, U$ has a g-radical supplement $V^{\prime}$ in $M$ with $V^{\prime} \leq V$, then we say $U$ has ample g-radical supplements in $M$. If every submodule of $M$ has ample gradical supplements in $M$, then $M$ is called an amply g-radical supplemented module. (See also [9].)

Clearly we can see that every amply g-radical supplemented module is g-radical supplemented. Since $\operatorname{Rad} M \leq \operatorname{Rad}_{g} M$ for every $R$-module $M$, every amply Rad-supplemented module is amply g-radical supplemented. Since every amply supplemented 
module is amply Rad-supplemented, every amply supplemented module is amply g-radical supplemented. Hollow and local modules are amply g-radical supplemented.

Proposition 3. Let $M=M_{1}+M_{2}+\cdots+M_{n}$. If $M_{i}$ is amply g-radical supplemented for every $i=1,2, \ldots, n$, then $M$ is $g$-radical supplemented.

Proof. Since $M_{i}$ is amply g-radical supplemented for every $i=1,2, \ldots, n, M_{i}$ is g-radical supplemented. Then by [3, Corollary 4], $M$ is g-radical supplemented.

Proposition 4. Let $M$ be an amply g-supplemented R-module. Then $M$ is amply g-radical supplemented.

Proof. Let $M=U+V$. Since $M$ is amply g-supplemented, $U$ has a g-supplement $V^{\prime}$ with $V^{\prime} \leq V$. Here $M=U+V^{\prime}$ and $U \cap V^{\prime} \ll_{g} V^{\prime}$. Since $U \cap V^{\prime} \ll_{g} V^{\prime}$, by Lemma $1, U \cap V^{\prime} \leq \operatorname{Rad}_{g} V^{\prime}$. Hence $V^{\prime}$ is a g-radical supplement of $U$ in $M$. Moreover, $V^{\prime} \leq V$. Hence $M$ is amply g-radical supplemented.

Proposition 5. Let $M$ be an R-module, $U_{1}, U_{2} \leq M$ and $M=U_{1}+U_{2}$. If $U_{1}$ and $U_{2}$ have ample g-radical supplements in $M$, then $U_{1} \cap U_{2}$ has also ample g-radical supplements in $M$.

Proof. Let $U_{1} \cap U_{2}+T=M$. Then by [2, Lemma 1.24], $M=U_{1}+U_{2} \cap T=$ $U_{2}+U_{1} \cap T$. Since $U_{1}$ and $U_{2}$ have ample g-radical supplements in $M$, then $U_{1}$ has a g-radical supplement $V_{1}$ with $V_{1} \leq U_{2} \cap T$ and $U_{2}$ has a g-radical supplement $V_{2}$ with $V_{2} \leq U_{1} \cap T$. Since $M=U_{1}+V_{1}$ and $V_{1} \leq U_{2}$, by Modular Law, $U_{2}=U_{2} \cap\left(U_{1}+V_{1}\right)=U_{1} \cap U_{2}+V_{1}$. Similarly we have $U_{1}=U_{1} \cap U_{2}+V_{2}$. Then $M=U_{1}+U_{2}=U_{1} \cap U_{2}+V_{2}+U_{1} \cap U_{2}+V_{1}=U_{1} \cap U_{2}+V_{1}+V_{2}$ and by Lemma 1, $U_{1} \cap U_{2} \cap\left(V_{1}+V_{2}\right)=U_{1} \cap\left(V_{1}+U_{2} \cap V_{2}\right)=U_{1} \cap V_{1}+U_{2} \cap V_{2} \leq \operatorname{Rad}_{g} V_{1}+\operatorname{Rad}_{g} V_{2} \leq$ $\operatorname{Rad}_{g}\left(V_{1}+V_{2}\right)$. Hence $V_{1}+V_{2}$ is a g-radical supplement of $U_{1} \cap U_{2}$ and since $V_{1}+V_{2} \leq$ $T, U_{1} \cap U_{2}$ has ample g-radical supplements in $M$.

Lemma 4. Every factor module of an amply g-radical supplemented module is amply g-radical supplemented.

Proof. Let $M$ be any amply g-radical supplemented module and $K \leq M$. Let $U / K \leq M / K$ and $M / K=U / K+V / K$ with $V / K \leq M / K$. Then $M=U+V$ and since $M$ is amply g-radical supplemented, there exists a g-radical supplement $T$ of $U$ with $T \leq V$. Then by [3, Lemma 8], $(T+K) / K$ is a g-radical supplement of $U / K$ in $M / K$. Moreover, $(T+K) / K \leq V / K$. Hence $U / K$ has ample g-radical supplements in $M / K$ and $M / K$ is amply g-radical supplemented.

Corollary 4. The homomorphic image of an amply g-radical supplemented module is amply g-radical supplemented.

Proof. Clear from Lemma 4. 
Lemma 5. Let $M$ be an R-module. If every submodule of $M$ is g-radical supplemented, then $M$ is amply g-radical supplemented.

Proof. Let $U \leq M$ and $M=U+V$ with $V \leq M$. By hypothesis, $V$ is g-radical supplemented and $U \cap V$ has a g-radical supplement $T$ in $V$. Here $V=U \cap V+T$ and $U \cap V \cap T \leq \operatorname{Rad}_{g} T$. Then $M=U+V=U+U \cap V+T=U+T$ and $U \cap T=$ $U \cap V \cap T \leq \operatorname{Rad}_{g} T$. Hence $U$ has ample g-radical supplements in $M$ and $M$ is amply g-radical supplemented.

Proposition 6. Let $R$ be any ring. Then every $R$-module is g-radical supplemented if and only if every $R$-module is amply g-radical supplemented.

Proof. ( $\Longrightarrow)$ Let $M$ be an $R$-module. Since every $R$-module is g-radical supplemented, every submodule of $M$ is g-radical supplemented. Then by Lemma 5, $M$ is amply g-radical supplemented, as desired.

$(\Longleftarrow)$ Clear.

Lemma 6. Let $M$ be a $\pi$-projective and g-radical supplemented module. Then $M$ is amply g-radical supplemented.

Proof. Let $M=U+V$ and $X$ be a g-radical supplement of $U$ in $M$. Since $M$ is $\pi$-projective and $M=U+V$, there exists an $R$-module homomorphism $f: M \rightarrow M$ such that $\operatorname{Im} f \subset V$ and $\operatorname{Im}(1-f) \subset U$. So, we have $M=f(M)+(1-f)(M)=$ $f(U)+f(X)+U=U+f(X)$. Suppose that $a \in U \cap f(X)$. Since $a \in f(X)$, there exists $x \in X$ such that $a=f(x)$. Since $a=f(x)=f(x)-x+x=x-(1-f)(x)$ and $(1-f)(x) \in U$, we have $x=a+(1-f)(x) \in U$. Thus $x \in U \cap X$ and so $a=f(x) \in$ $f(U \cap X)$. Therefore we have $U \cap f(X) \leq f(U \cap X) \leq f\left(\operatorname{Rad}_{g} X\right) \leq \operatorname{Rad}_{g} f(X)$. This means that $f(X)$ is a g-radical supplement of $U$ in $M$. Moreover, $f(X) \subset V$. Therefore $M$ is amply g-radical supplemented.

Corollary 5. If $M$ is a projective and g-radical supplemented module, then $M$ is an amply g-radical supplemented module.

Proof. Clear from Lemma 6.

Lemma 7. Let $M=M_{1} \oplus M_{2} \oplus \cdots \oplus M_{n}$. If $M_{i}$ is projective and g-radical supplemented for every $i=1,2, \ldots, n$, then $M$ is amply g-radical supplemented.

Proof. Since $M_{i}$ projective for every $i=1,2, \ldots, n$, by [2, 4.3], $M$ is projective. Since $M_{i}$ is g-radical supplemented for every $i=1,2, \ldots, n$, by [3, Corollary 4], $M$ is g-radical supplemented. Then by Corollary 5, $M$ is amply g-radical supplemented, as desired.

Lemma 8. Let $M$ be a projective and g-radical supplemented module. Then every finitely $M$-generated module is amply g-radical supplemented.

Proof. Let $N$ be a finitely $M$-generated $R$-module. Then there exist a finite index set $\Lambda$ and an $R$-module epimorphism $f: M^{(\Lambda)} \longrightarrow N$. Since $M$ is projective and 
g-radical supplemented, by Lemma $7, M^{(\Lambda)}$ is amply g-radical supplemented. Then by Corollary 4, $N$ is amply g-radical supplemented.

Proposition 7. Let $R$ be any ring. The following assertions are equivalent.

(i) ${ }_{R} R$ is g-radical supplemented.

(ii) ${ }_{R} R$ is amply $g$-radical supplemented.

(iii) ${ }_{R} R^{(\Lambda)}$ is g-radical supplemented for every finite index set $\Lambda$.

(iv) Every finitely generated $R$-module is g-radical supplemented.

(v) Every finitely generated $R$-module is amply g-radical supplemented.

Proof. (i) $\Longleftrightarrow$ (ii) Clear from Corollary 5 , since ${ }_{R} R$ is projective.

(i) $\Longleftrightarrow$ (iii) Clear from [3, Corollary 4].

(i) $\Longleftrightarrow$ (iv) Clear from [3, Corollary 6].

(i) $\Longleftrightarrow$ (v) Clear from Lemma 8 .

\section{REFERENCES}

[1] G. F. Birkenmeier, F. T. Mutlu, C. Nebiyev, N. Sokmez, and A. Tercan, "Goldie*supplemented modules," Glasgow Mathematical Journal, vol. 52A, pp. 41-52, 2010, doi: 10.1017/s0017089510000212.

[2] J. Clark, C. Lomp, N. Vanaja, and R. Wisbauer, Lifting Modules: Supplements and Projectivity in Module Theory (Frontiers in Mathematics), 2006th ed. Basel: Birkhäuser, 8 2006. doi: 10.1007/3-7643-7573-6.

[3] B. Koşar, C. Nebiyev, and A. Pekin, "A generalization of g-supplemented modules," Miskolc Mathematical Notes, vol. 20, no. 1, pp. 345-352, 2019, doi: 10.18514/mmn.2019.2586.

[4] B. Koşar, C. Nebiyev, and N. Sökmez, "G-supplemented modules," Ukrainian Mathematical Journal, vol. 67, no. 6, pp. 975-980, Nov. 2015, doi: 10.1007/s11253-015-1127-8.

[5] C. Nebiyev and H. H. Ökten, "Weakly g-supplemented modules," European Journal of Pure and Applied Mathematics, vol. 10, no. 3, pp. 521-528, 2017.

[6] C. Nebiyev and A. Pancar, "On supplement submodules," Ukrainian Mathematical Journal, vol. 65, no. 7, pp. 1071-1078, Dec. 2013, doi: 10.1007/s11253-013-0842-2.

[7] C. Nebiyev, "g-radical supplemented modules," in Antalya Algebra Days XVII, Şirince, İzmir, Turkey, 2015.

[8] C. Nebiyev, "On a generalization of supplement submodules," International Journal of Pure and Applied Mathematics, vol. 113, no. 2, pp. 283-289, 2017, doi: 10.12732/ijpam.v113i2.8.

[9] C. Nebiyev, "Amply g-radical supplemented modules," in X International Conference of the Georgian Mathematical Union, Batumi, Georgia, 2019.

[10] C. Nebiyev and N. Sökmez, "Beta g-star relation on modules," European Journal of Pure and Applied Mathematics, vol. 11, no. 1, pp. 238-243, Jan. 2018, doi: 10.29020/nybg.ejpam.v11i1.2741.

[11] N. Sökmez, B. Koşar, and C. Nebiyev, "Genelleştirilmiş küçük alt modüller," in XIII. Ulusal Matematik Sempozyumu. Kayseri: Erciyes Üniversitesi, 2010.

[12] Y. Wang and N. Ding, "Generalized supplemented modules," Taiwanese Journal of Mathematics, vol. 10, no. 6, Dec. 2006, doi: 10.11650/twjm/1500404577.

[13] R. Wisbauer, Foundations of Module and Ring Theory. Philadelphia: Gordon and Breach, 1991. doi: $10.1201 / 9780203755532$.

[14] D. X. Zhou and X. R. Zhang, "Small-essential submodules and morita duality," Southeast Asian Bulletin of Mathematics, vol. 35, pp. 1051-1062, 2011. 
Authors' addresses

\section{Berna Koşar}

Uskudar University, Department of Health Management, Üsküdar, İstanbul, Turkey

E-mail address: bernak@omu.edu.tr, berna.kosar@uskudar.edu.tr

Ayten Pekin

(Corresponding author) Istanbul University, Department of Mathematics, Vezneciler, İstanbul, Turkey

E-mail address: aypekin@istanbul.edu.tr, aspekin@hotmail.com 